\title{
VOLUME AND TEMPERATURE DEPENDENCE OF THE TRANSVERSE CHARGE AND THE IONICITY OF TETRAHEDRAL SEMICONDUCTORS
}

\author{
D. Olego, M. Cardona and P. Vog $1^{*}$ \\ Max-Planck-Institut für Festkörperforschung, Stuttgart, F.R.G. \\ * University of Graz, Austria
}

\begin{abstract}
The transverse charge $e^{*}$ and thus the ionicity of most tetrahedral semiconductors (e.g. GaAs) decreases with decreasing volume. We have measured the volume dependence of $e_{m}^{*}$ in $3 C-S i C$ and found din $e_{\mathrm{T}}^{*} / \mathrm{dlnV}=-0.67$, with a sign opposite to the usual one. These resufts have been interpreted with pseudopotential calculations of $e_{\mathrm{T}}^{*}$ vs. volume. Semiempirical bond orbital models, (e.g. Harrison), are not able to explain this anomalous behavior of $e_{\mathrm{T}}^{*}$. We also report the temperature dependence of $\mathrm{e}_{\mathrm{T}}^{*}$ - $\mathrm{E}_{\mathrm{f}}$ interpret $^{\mathrm{T}}$ it with pseudopotential theory $\left(\mathrm{de}_{\mathrm{T}}^{*} / \mathrm{dT}=-9 . \mathrm{O}(5) \times 1 \mathrm{~J}^{-6} \mathrm{~K}^{-1}\right)$.
\end{abstract}

1. Introduction. - The LO-TO splitting of the Raman phonons can be used to obtain the corresponding Born transverse effective charges $e_{T}^{*}$. Measurements in a diamond anvil cell yield the dependence of $e_{T}^{*}$ on volume. They have been performed for several III-V and II-VI compounds $(1,2)$ and lead, in all cases, to a decrease in $e_{T}^{*}$ with pressure which corresponds to a decrease in ionicity. These results have been interpreted with semiempirical bond models (3) as well as with "microscopic" pseudopotential calculations (4). Here we extend this work to 3C-SiC (zincblende). We found that, contrary to the other materials mentioned, $e_{T}^{*}$ in $3 C-s i c$ increases with increasing pressure. This anomaly can also be accounted for with pseudopotential theory. We also report the temperature dependence of $e_{T}^{*}$. It can be explained with pseudopotential theory by multiplying the form factors by Debye-waller terms.

2. Results. - Figure 1 displays typical spectra of the To and Lo phonons of 3C-SiC in a way which reveals the increase of the LO-TO splitting with pressure (p). The variation of the frequency of these phonons with $\mathrm{p}$ is shown in Fig. 2. The corresponding variation of lattice constant a, calculated with Murnaghan's equation (1) using $\mathrm{B}_{\mathrm{O}}=322$ Gpa and $\mathrm{B}^{\prime}{ }_{\mathrm{O}}=3.43$ (average values of $\mathrm{Si}$ and diamond), is also given as an abscissa. We find from Fig. 2 the Grüneisen parameters $\gamma_{\text {TO }}=1.56(1), \gamma_{\mathrm{LO}}=$ 1.55(1). The increase in the Lo-TO splitting can also be seen in Fig.2. The " $\gamma$ " corresponding to LO-TO is $\gamma_{\text {LO-TO }}=1.52(5)$. The charge $e_{T}^{*}$ is given by:

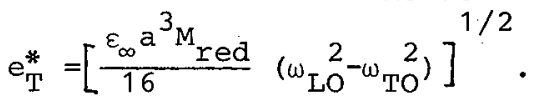


where $M_{r e d}$ is the reduced mass of the unit cell. From the variation of ${ }^{\omega_{L O}}{ }^{-\omega_{\text {TO }}}$ with volume we evaluate with Eq. 1 the dependence of $\mathrm{e}_{\mathrm{T}}^{*}$ on volume using the volume coefficient of the ir dielectric constant $\mathrm{d} l n \varepsilon_{\infty} / \mathrm{d} \ln \mathrm{a}=1.8$ (the average of $\mathrm{Si}$ and diamond). The results are displayed in Fig. 3. A linear fit yields:

$$
\mathrm{e}_{\mathrm{T}}^{*}=2.697(4)-5.45(10) \frac{\Delta \mathrm{a}}{\mathrm{a}_{\mathrm{o}}} \text {. }
$$

From Eq. (2) we calculate $\gamma_{e_{T}^{*}}^{*}=-d \ln e_{\mathrm{T}}^{*} / \mathrm{d} l n \mathrm{~V}=0.67(2)$.

The charge $e_{T}^{*}$ can be refated to the pseudopotential form factors

$\mathrm{V}(\mathrm{G})$ of $\mathrm{Si}$ and $\mathrm{C}$ with the approximate expression (4):

$$
e_{T}^{*}=-4 \frac{v_{S i}^{2}(3)-v_{C}^{2}(3)}{v_{S i(3)}^{2}+v_{C}^{2}(3)}
$$

We use form factors similar to those of (5) and interpolate them smooth$1 y$, so as to obtain the derivatives of $v(G)$ with respect to $G$. By differentiating Eq. (3) with respect to a we obtain the dashed line of Fig. 3 which represents reasonably well the experiment. The squares in this figure give the results of a full numerical pseudopotential calculation $(1,4)$. The circles represent bond orbitals model calculations (3): They strongly deviate from the experiment.

The reason for the difference in the volume dependence of $e_{T}^{*}$ between $3 \mathrm{C}-\mathrm{SiC}$ and the other zincblende-semiconductors is to be sought in the different origin of their ionicity. In Sic the ionicity is related to the large difference in the radii of $S i$ and $C$. Consequently part of the electronic charge of Si lies in the wigner-Seitz sphere of $\mathrm{C}$. A decrease in the lattice constant pushes more of this charge into the WS sphere of $C$ and thus increases the ionicity and $e_{T}^{*}$.

For completeness we show in Fig. 4 the experimental points obtained for $e_{\mathrm{T}}^{*}$ at several temperatures together with a least squares fit: $\left(\mathrm{de}_{\mathrm{T}}^{*} / \mathrm{dT}\right)_{\mathrm{p}}=-9 . \mathrm{O}(5) \times 10^{-6} \mathrm{~K}^{-1}$. This coefficient is very small due to a compensation between the thermal expansion and the explicit temperature effects. The latter can be calculated with pseudopotential theory using Debye-waller factors (6)..We find:

$$
\left(\frac{\partial e_{T}^{*}}{\partial \cdot T}\right)_{V}=\begin{array}{ll}
1.28(50) \times 10^{-5} K^{-1} & \text { (experiment) } \\
2.3 \times 10^{-5} \mathrm{~K}^{-1} & \text { (theory). }
\end{array}
$$

We thank W.J. Choyke for supplying us the samples of $3 C-S i C$.

\section{References}

1. R. Trommer, H. Müller, M. Cardona, and P. Vogl, Phys. Rev. B21, 4869 (1980)

2. B.A. Weinstein, Solid State Comm. 24, 595 (1977)

3. W. Harrison, Electronic Structure and Properties of solids,

(Freeman, San Francisco, 1980) 
4. P. Vogl, J. Phys. C 11, 251 (1978)

5. H.G. Junginger and W. van Haeringer, Phys. Stat. Sol.37,709 (1970)

6. D. Olego and M. Cardona, to be published

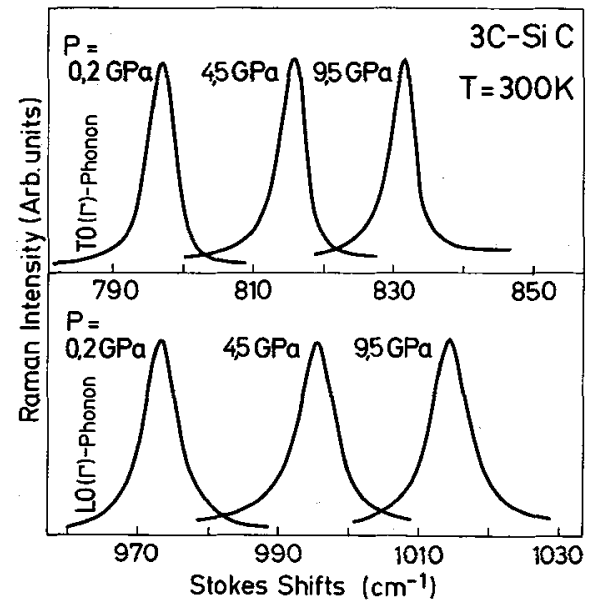

Fig. 1 LO and TO Raman lines of 3C-SiC at several pressures.

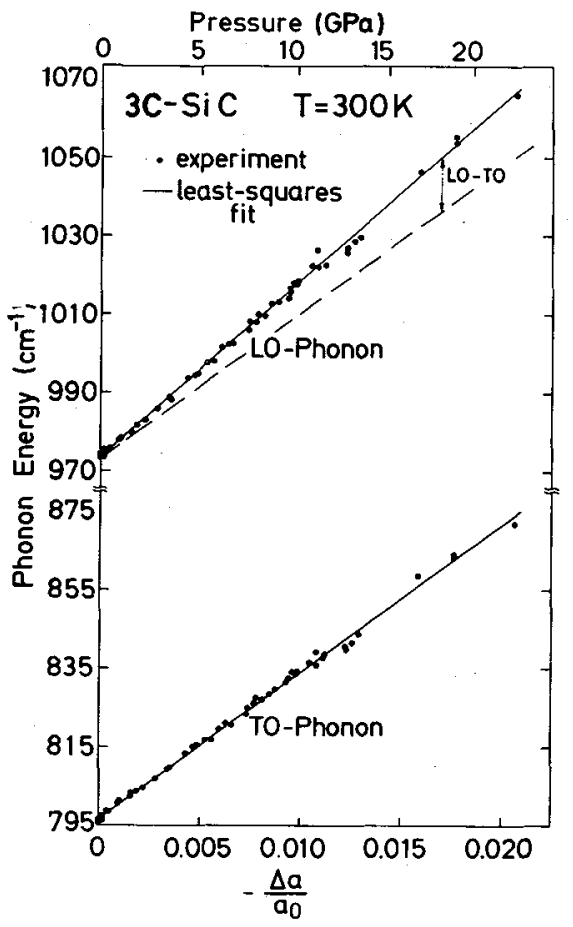

Fig. 2 Dependence of the LO and TO frequencies on pressure and lattice constant for $3 \mathrm{C}-\mathrm{SiC}$

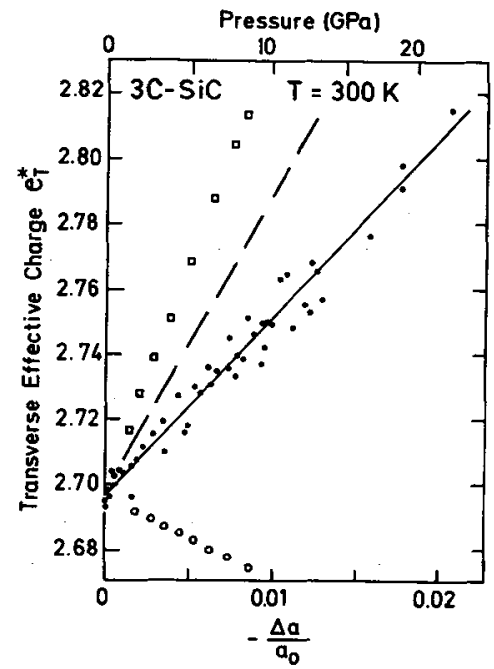

Fig. 3 Dependence of $e_{T}^{*}$ on pressure and lattice constant measured for 3C-SiC (points). Dashed line: calculation with $\mathrm{Eq}$. 3. Circles: bond orbital model. Squares: Full pseudopotential calculation.

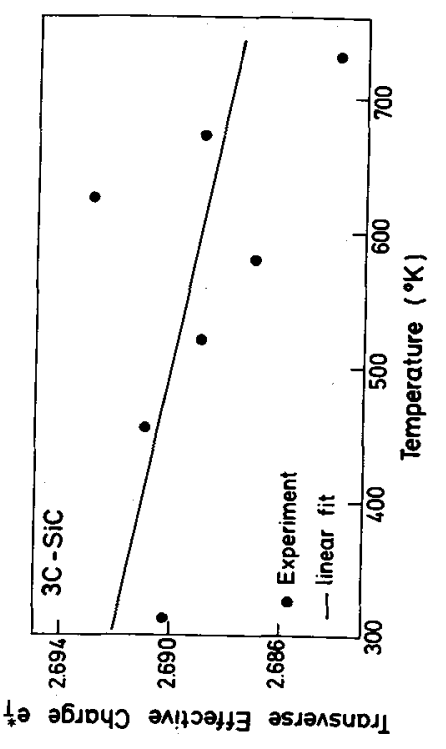

Fig. 4 Measured temperature dependence of $e^{*}$ for 3c-Sic (points). Iine: least squares fit. 